\title{
ON DIMENSION SUBGROUPS
}

\author{
BY \\ GERALD LOSEY(1)
}

1. Introduction. Let $G$ be a group and $R$ a commutative ring with identity element. The group ring $\Gamma$ of $G$ over $R$ consists of all formal sums $\sum \alpha(g) g$ where $g \in G, \alpha(g) \in R$ and $\alpha(g)=0$ except for a finite number of $g$. The operations of addition, multiplication and multiplication by elements of $R$ are defined in the natural manner. Thus, $\Gamma$ is a free $R$-module with a multiplication induced by the group operation in $G$. The set of all those sums $\sum \alpha(g) g$ for which $\sum \alpha(g)=0$ is an ideal $\Delta$ of $\Gamma$, called the fundamental ideal of $\Gamma$.

If $\Lambda$ is any ideal of $\Gamma$, then

$$
\mathfrak{S}(\Lambda)=\{g \in G: g \equiv 1 \bmod \Lambda\}
$$

is a normal subgroup of $G$. The subgroup $D_{n}=\mathfrak{S}\left(\Delta^{n}\right)$ of $G$ is called the $n$th dimension subgroup modulo $R$ of $G$. These subgroups are modeled in their construction on the dimension subgroups of free groups defined by Magnus [7] and Zassenhaus [8]. Lazard [6] has shown that the dimension subgroups modulo $Z_{p}$, the ring of integers modulo the prime $p$, are characterized by precisely the same properties, discovered by Zassenhaus, which characterize the dimension subgroups modulo $Z_{p}$ of a free group. Our main result will show that a similar situation holds for the construction of Magnus: If $F$ is a free group then the dimension subgroups of Magnus are identical with the dimension subgroups modulo $Z$, the ring of rational integers, as described above. Namely, they are exactly the subgroups of the lower central series of $F$. This result has previously been proved by Fox [2].

This leads to the conjecture (Cohn [1], Lazard [6]) that the dimension subgroups modulo $Z$ of any group $G$ are exactly the subgroups of the lower central series of $G$. The principal aim of this paper is to verify this conjecture. Using this result, it will be shown that the factors of the lower central series may be calculated directly from the group ring.

The author wishes to express his appreciation to Professor Roger Lyndon for his aid and encouragement.

2. The group ring and the fundamental ideal. Let $\eta: G \rightarrow H$ be a (group) homomorphism. $\eta$ can be extended to an $R$-homomorphism of $\Gamma_{G}$ into $\Gamma_{H}$ by setting

$$
\bar{\eta}\left[\sum \alpha(g) g\right]=\sum \alpha(g) \eta(g)
$$

Jennings [3] has shown the following:

Received by the editors March 12, 1960.

(1) This paper is based, in part, on the author's doctoral dissertation, University of Michigan, 1958. 
LEMma 2.1. If $\eta: G \rightarrow H$ is a homomorphism with kernel $K$, then $\bar{\eta}: \Gamma_{G} \rightarrow \Gamma_{H}$ is an $R$-homomorphism with kernel $I(K)$, where $I(K)$ is the ideal of $\Gamma_{G}$ generated by all elements $k-1$ for $k \in K$.

LEMma 2.2. If $o: G \rightarrow 1$ is the trivial homomorphism then its extension $\bar{o}: \Gamma \rightarrow R$ has kernel $\Delta$. Thus $\Delta$ is an ideal. The elements $g-1$ for all $g \neq 1$ in $G$ are an $R$-basis for $\Delta$.

Proof. The first statement follows from Lemma 2.1 and the definition of $\Delta$. Now, suppose $\sum \alpha(g) g \in \Delta$. Then $\sum \alpha(g)=0$ and so

$$
\sum \alpha(g) g=\sum \alpha(g) g-\sum \alpha(g)=\sum \alpha(g)(g-1) .
$$

Hence, the $g-1 \operatorname{span} \Delta$. On the other hand, if $\sum \alpha(g)(g-1)=0$ then $\sum \alpha(g) g$ $-\left(\sum \alpha(g)\right) \cdot 1=0$ and so $\alpha(g)=0$ for each $g$. Therefore, the $g-1$ are linearly independent over $R$.

TheOREM 2.3. Let $\bar{o}: \Gamma \rightarrow R$ be the canonical homomorphism sending $\sum \alpha(g) g$ onto $\sum \alpha(g)$. Let $\eta: \Gamma \rightarrow R$ be any other $R$-epimorphism. Then there is an automorphism $\tau$ of $\Gamma$ such that $\eta=\bar{o} \cdot \tau$.

Proof. Since $\eta$ is an epimorphism, $\eta(1)=1$. Hence, for any $g \in G, \eta(g) \eta\left(g^{-1}\right)$ $=1$ and so $\eta(g)^{-1}$ exists. Define $\tau: \Gamma \rightarrow \Gamma$ by

$$
\tau\left[\sum \alpha(g) g\right]=\sum \alpha(g) \eta(g) g \text {. }
$$

It is easily verified that $\tau$ is an endomorphism of $\Gamma$. Suppose $\sum \alpha(g) \eta(g) g=0$; then $\alpha(g) \eta(g) \doteq 0$ for each $g$. Since $\eta(g)^{-1}$ exists, $\alpha(g)=0$ for each $g$ and so $\sum \alpha(g) g=0$. Thus the kernel of $\tau$ is (0). Given any $\sum \alpha(g) g \in \Gamma$, note that

$$
\tau\left[\sum \alpha(g) \eta(g)^{-1} g\right]=\sum \alpha(g) g
$$

and, hence, $\tau$ is onto. Therefore, $\tau$ is an automorphism. Moreover,

$$
\bar{o} \cdot \tau\left[\sum \alpha(g) g\right]=\bar{o}\left[\sum \alpha(g) \eta(g) g\right]=\sum \alpha(g) \eta(g)=\eta\left[\sum \alpha(g) g\right] .
$$

Therefore, $\bar{o} \cdot \tau=\eta$.

COROLlaRy 2.4. Let $\eta: \Gamma \rightarrow R$ be any $R$-epimorphism with kernel $\Lambda$. Then $\Lambda \cong \Delta$.

Proof. Let $\tau$ be the automorphism defined above. If $\sum \alpha(g) g \in \Lambda$, then $\sum \alpha(g) \eta(g)=0$, or, in other words,

$$
\sum \alpha(g) \eta(g) g=\tau\left(\sum \alpha(g) g\right) \in \Delta .
$$

Hence, $\tau(\Lambda) \subseteq \Delta$. Conversely, let $\sum \alpha(g) g \in \Delta$. Then $\sum \alpha(g) \eta(g)^{-1} g \in \Lambda$ since

$$
\eta\left(\sum \alpha(g) \eta(g)^{-1} g\right)=\sum \alpha(g)=0 .
$$

Also, $\tau\left(\sum \alpha(g) \eta(g)^{-1} g\right)=\sum \alpha(g) g$. Therefore, $\tau(\Lambda) \supseteq \Delta$. Hence, $\tau(\Lambda)=\Delta$ and so $\Lambda$ and $\Delta$ are isomorphic. 
A consequence of this result is that $\Delta$ is determined up to isomorphism by the structure of $\Gamma$ as an $R$-ring, without any reference to the group $G$. In particular, if $G$ and $H$ are groups with isomorphic group rings then they also have isomorphic fundamental ideals.

3. The dimension subgroups. As indicated in the introduction, we define

$$
D_{n}=D_{n}(G ; R)=\left\{g \in G: g \equiv 1 \bmod \Delta^{n}\right\} .
$$

It is easily verified that $D_{n}$ is a normal subgroup of $G$. Since $\Delta \supseteq \Delta^{2} \supseteq \Delta^{3} \supseteq \cdots \cdots$, the dimension subgroups form a descending series,

$$
G=D_{1} \supseteq D_{2} \supseteq D_{3} \supseteq \cdots \cdot
$$

This sequence is called the dimension series $\bmod R$ of $G$.

In the sequel, the notation $(h, k)=h^{-1} k^{-1} h k$ is used. By $(H, K)$ is meant the subgroup of $G$ generated by all commutators $(h, k), h \in H, k \in K$. The lower central series is defined $G=G_{1}, G_{n+1}=\left(G_{n}, G\right)$. A descending sequence of subgroups $\left\{H_{i}\right\}$ is said to be a descending central series if $\left(H_{i}, G\right) \subseteq H_{i+1}$ for every $i$. If $\left\{H_{i}\right\}$ is a descending central series of $G$, then $H_{i} \supseteq G_{i}$ for every $i$.

THEOREM 3.1. The dimension subgroups of $G$ form a descending central series of fully invariant subgroups of $G$. Moreover, $\left(D_{n}, D_{m}\right) \subseteq D_{n+m}$. Hence, $D_{n} \supseteq G_{n}$ for every $n$.

Proof. Verification of $\left(D_{n}, D_{m}\right) \subseteq D_{n+m}$ will show that the series is central. $\left(D_{n}, D_{m}\right)$ is generated by all commutators $\left(g_{n}, g_{m}\right), g_{n} \in D_{n}, g_{m} \in D_{m}$. Since $g_{n}-1 \in \Delta^{n}$ and $g_{m}-1 \in \Delta^{m}$, the element

$$
g^{n} g_{m}-g_{m} g_{n}=\left(g_{n}-1\right)\left(g_{m}-1\right)-\left(g_{m}-1\right)\left(g_{n}-1\right)
$$

belongs to $\Delta^{n+m}$. Therefore

$$
\begin{aligned}
\left(g_{n}, g_{m}\right) & =g_{n}^{-1} g_{m}^{-1} g_{n} g_{m} \\
& =1+g_{n}^{-1} g_{m}^{-1}\left(g_{n} g_{m}-g_{m} g_{n}\right) \\
& \equiv 1 \bmod \Delta^{n+m} .
\end{aligned}
$$

Hence, $\left(g_{n}, g_{m}\right) \in D_{n+m}$.

Let $g$ be any element of $D_{n}$. Then $g$ may be expressed in the form

$$
g=1+\sum_{i=1}^{k} \alpha_{i} \prod_{j=1}^{n}\left(g_{i j}-1\right) .
$$

Let $\gamma$ be any endomorphism of $G$. Applying its extension $\bar{\gamma}$ to both sides of the above equation gives

$$
\gamma(g)=1+\sum_{i=1}^{k} \alpha_{i} \prod_{j=1}^{n}\left(\gamma\left(g_{i j}\right)-1\right),
$$

and so $\gamma(g) \equiv 1 \bmod \Delta^{n}$ and, thus, $\gamma(g) \in D_{n}$. Therefore, $D_{n}$ is fully invariant. 
Jennings [5] has proved the following result for the dimension subgroups of a finite $p$-group modulo a field of characteristic $p$. His proof is easily extendible to the general case.

THEOREM 3.2. Let $H$ be any normal subgroup of $G$ and $n$ the largest integer such that $D_{n} \supseteq H$. Then the first $n$ dimension subgroups of $G / H$ are

$$
G / H=D_{1} / H \supseteq D_{2} / H \supseteq \cdots \supseteq D_{n} / H \text {. }
$$

It is unknown if dimension subgroups, in general, are preserved under homomorphism.

4. Some isomorphism theorems. The mapping

$$
i: D_{n} \rightarrow \Delta^{n} / \Delta^{n+1} \text {, }
$$

defined by $i(g)=(g-1)+\Delta^{n+1}$, is well defined since $g \in D_{n}$ if and only if $g-1 \in \Delta^{n}$. If $g$ and $h$ are in $D_{n}$, then

$$
\begin{aligned}
i(g h) & =(g h-1)+\Delta^{n+1} \\
& =(g-1)+(h-1)+(g-1)(h-1)+\Delta^{n+1} \\
& =(g-1)+(h-1)+\Delta^{n+1}=i(g)+i(h) .
\end{aligned}
$$

Consequently, $i$ is a homomorphism of $D_{n}$ into the additive group structure of $\Delta^{n} / \Delta^{n+1}$. The kernel of $i$ is clearly $D_{n+1}$. Thus, we have proved

Theorem 4.1. There is an imbedding $i$ of $D_{n} / D_{n+1}$ in the additive group structure of $\Delta^{n} / \Delta^{n+1}$.

COROLLARY 4.2. If the ring $R$ has characteristic $m$, then the order of every element of $D_{n} / D_{n+1}$ divides $m$. In other words, $D_{n}^{m} \subseteq D_{n+1}$, where $D_{n}^{m}$ denotes the subgroup of $G$ generated by all mth powers of elements from $D_{n}$.

Note that the subgroup $\tilde{\imath}\left(D_{n} / D_{n+1}\right)$ of $\Delta^{n} / \Delta^{n+1}$ does not necessarily admit operators from $R$. However, if we restrict ourselves to the case $R=Z_{m}$, the ring of integers $\bmod m$ (or just the ring of ordinary integers if $m=0$ ), this is no longer the case, i.e., $\tilde{\imath}\left(D_{n} / D_{m}\right)$ is a $Z_{m}$-submodule of $\Delta^{n} / \Delta^{n+1}$. In this case, we shall speak of the dimension subgroups modulo $m$.

Assume $R=Z_{m}$. The image of $D_{n}$ under $i$ will be the submodule of $\Delta^{n} / \Delta^{n+1}$ generated by all cosets $(g-1)+\Delta^{n+1}, g \in D_{n}$. Denote the submodule of $\Gamma$ generated by all $g-1, g \in D_{n}$, by $M_{n}$. Then the image of $D_{n}$ is

$$
\left(M_{n}+\Delta^{n+1}\right) / \Delta^{n+1} \text {. }
$$

If $h-1$ is one of the generators of $M_{n}$ and $\sum \alpha(g) g \in \Gamma$, then

$$
\begin{aligned}
(h-1) \sum \alpha(g) g & =\sum \alpha(g)(h-1) g \\
& =\sum \alpha(g)(h-1)(g-1)+\left(\sum \alpha(g)\right)(h-1) \\
& \equiv\left(\sum \alpha(g)\right)(h-1) \bmod \Delta^{n+1} .
\end{aligned}
$$


Thus, $M_{n} \cdot \Gamma \subseteq M_{n}+\Delta^{n+1}$. Similarly, $\Gamma \cdot M_{n} \subseteq M_{n}+\Delta^{n+1}$. It follows that $M_{n}+\Delta^{n+1}$ is an ideal of $\Gamma$. Therefore, $M_{n}+\Delta^{n+1}$ will contain the ideal of $\Gamma$ generated by $M_{n}$, namely $I\left(D_{n}\right)$ (see Lemma 2.1). Hence,

$$
I\left(D_{n}\right) \subseteq M_{n}+\Delta^{n+1} \text { and } M_{n} \subseteq I\left(D_{n}\right),
$$

and so $M_{n}+\Delta^{n+1}=I\left(D_{n}\right)+\Delta^{n+1}$.

TheOREM 4.3. Let $\left\{D_{i}\right\}$ be the dimension series $\bmod m$ of $G$. Then

$$
D_{n} / D_{n+1} \cong\left(I\left(D_{n}\right)+\Delta^{n+1}\right) / \Delta^{n+1} .
$$

Define the Lie product in $\Gamma$ in the usual manner: $[x, y]=x y-y x$. Set $\Delta^{(1)}=\Delta, \Delta^{(n+1)}=\left[\Delta^{(n)}, \Delta\right]$. It is clear that $\Delta^{(n)} \subseteq \Delta^{n}$. Extend the use of the bracket symbol to include $[g]=g-1$ for elements of $G$ and extend it to $\Gamma$ by linearity, i.e.,

$$
\left[\sum \alpha(g) g\right]=\sum \alpha(g)(g-1) .
$$

A Lie product in $\Gamma$ is called left normed if it has the form

$$
\left[\left[\cdots\left[\left[x_{1}, x_{2}\right], x_{3}\right], \cdots\right], x_{n}\right] \text {. }
$$

Such a product shall be written, more simply, as $\left[x_{1}, x_{2}, \cdots, x_{n}\right]$. It is clear from the definition of $\Delta^{(n)}$ and from the bilinearity of the Lie product that all such products with $x_{i}=g_{i} \in G$ span $\Delta^{(n)}$.

Let $\left(g_{1}, g_{2}, \cdots, g_{n}\right)$ denote the left normed commutator

$$
\left(\left(\cdots\left(\left(g_{1}, g_{2}\right), g_{3}\right), \cdots\right), g_{n}\right) \text {. }
$$

LEMMA 4.4.

$$
\left(g_{1}, \cdots, g_{n}\right) \equiv 1+\left[g_{1}, \cdots, g_{n}\right] \bmod \Delta^{n+1} .
$$

Proof. The lemma is clearly true for $n=1$. Assume it is true for $n$. Then, if $c_{n}=\left(g_{1}, \cdots, g_{n}\right)$ and $d_{n}=\left[g_{1}, \cdots, g_{n}\right]$, by the induction hypothesis $c_{n}=1+d_{n}+\delta$, where $\delta \in \Delta^{n+1}$. Hence,

$$
\begin{aligned}
\left(g_{1}, \cdots, g_{n}, g_{n+1}\right) & =c_{n}^{-1} g_{n+1}^{-1} c_{n} g_{n+1} \\
& =1+c_{n}^{-1} g_{n+1}^{-1}\left(c_{n} g_{n+1}-g_{n+1} c_{n}\right) \\
& =1+c_{n}^{-1} g_{n+1}^{-1}\left\{\left(1+d_{n}+\delta\right) g_{n+1}-g_{n+1}\left(1+d_{n}+\delta\right)\right\} \\
& =1+c_{n}^{-1} g_{n+1}^{-1}\left[g_{1}, \cdots, g_{n}, g_{n+1}\right]+\bar{\delta},
\end{aligned}
$$

where $\bar{\delta} \in \Delta^{n+2}$. Since $c_{n}^{-1} g_{n+1}^{-1} \in G$, it may be replaced by $1+\beta$, where $\beta \in \Delta$. Then both $\bar{\delta}$ and $\beta\left[g_{1}, \cdots, g_{n+1}\right]$ belong to $\Delta^{n+2}$. Therefore,

$$
\left(g_{1}, \cdots, g_{n+1}\right) \equiv 1+\left[g_{1}, \cdots, g_{n+1}\right] \bmod \Delta^{n+2} .
$$


Since $G_{n}$ is generated by the left normed commutators of weight $n$, the mapping $i$ defined above maps $G_{n}$ onto $\left(\Delta^{(n)}+\Delta^{n+1}\right) / \Delta^{n+1}$.

Theorem 4.5. Let $\left\{D_{i}\right\}$ be the dimension series $\bmod m$. Then,

$$
G_{n} D_{n+1} / D_{n+1} \cong\left(\Delta^{(n)}+\Delta^{n+1}\right) / \Delta^{n+1} \text {. }
$$

COROLlary 4.6. If $G$ is any group in which the dimension series $\bmod m$ coincides with the lower central series, then

$$
G_{n} / G_{n+1} \cong\left(\Delta^{(n)}+\Delta^{n+1}\right) / \Delta^{n+1} .
$$

5. Dimension subgroups modulo $Z$. In this section it will be shown that the dimension subgroups modulo the ring $Z$ of rational integers of a group $G$ coincide with the subgroups of the lower central series of $G$. We first deal with the case of a finitely generated group.

THEOREM 5.1. Let $G$ be a finitely generated group and $\Gamma$ the group ring of $G$ over $Z$. Let $G=G_{1} \supseteq G_{2} \supseteq G_{3} \supseteq \ldots$ be the lower central series of $G$. Then there is a sequence

$$
\Delta=\Lambda_{1} \supseteq \Lambda_{2} \supseteq \Lambda_{3} \supseteq \cdots
$$

of ideals of $\Gamma$ having the properties

(i) $g \in G_{i}$ if and only if $g-1 \in \Lambda_{i}$

(ii) $\Delta^{i} \subseteq \Lambda_{i}$.

Therefore, $D_{i} \subseteq G_{i}$ and consequently, since $\left\{D_{i}\right\}$ is a descending central series, $D_{i}=G_{i}$.

The proof of this theorem will require the establishment of several preliminary results.

The sequence $\left\{G_{i}\right\}$ induces a weight function $w$ on the elements of $G$ :

$$
w(g)=\operatorname{Max}\left\{n: g \in G_{n}\right\}
$$

with the agreement that $w(g)=\infty$ if $g$ belongs to all $G_{n}$. If $(g, h)$ is a commutator, then

$$
w((g, h)) \geqq w(g)+w(h),
$$

since $\left(G_{i}, G_{j}\right) \subseteq G_{i+j}$. For any element $g \in G$, let $\bar{g}$ denote the coset $g G_{w(g)+1}$.

Since we are assuming that $G$ is finitely generated, so is each of the factor groups $G_{i} / G_{i+1}$, (see, e.g., M. Hall [3]). Hence, $G_{i} / G_{i+1}$ is a finitely generated abelian group and so has a basis of cosets $\bar{x}_{i(j)}$. From each of these basis cosets pick a representative $x_{i(j)}$. To this collection adjoin the group elements $x_{i(j)}^{-1}$ for each $x_{i(j)}$ for which $\bar{x}_{i(j)}$ has infinite order. Denote the resulting collection by $\phi_{i}$. Well order $\phi_{i}$ in such a manner that $x_{i(j)}^{-1}$, when it is present, is the immediate successor of $x_{i(j)}$. Let $\phi=U_{\phi_{i}}$. Extend the well ordering of the $\phi_{i}$ to $\phi$ by putting each member of $\phi_{i}$ ahead of each member of $\phi_{i+1}$. Let $I$ be the set of all ordinal numbers less than the order type of $\phi$; then $\phi$ may be indexed by $I$ in an order preserving manner: $\phi=\left\{x_{\alpha}\right\}_{\alpha \in I}$. 
For a fixed integer $n$, each element $g \in G$ has a unique representation

$$
g \equiv x_{\alpha(1)}^{e(1)} x_{\alpha(2)}^{e(2)} \cdots x_{\alpha(k)}^{e(k)} \bmod G_{n+1},
$$

where

(i) $\alpha(1)<\alpha(2)<\cdots<\alpha(k)$,

(ii) $w\left(x_{\alpha(j)}\right) \leqq n$,

(iii) $0<e(j)<$ order of $\bar{x}_{\alpha(j)}$,

(iv) not both $x_{\alpha(j)}$ and $x_{\alpha(j)}^{-1}$ occur.

We shall, for the time being, assume that $G_{n+1}=1$. Thus, the congruence (1) becomes an equation.

Consider the set of all families $\left(h_{\alpha}\right)_{\alpha \in I}$ of non-negative integers such that $h_{\alpha}=0$ except for finitely many $\alpha \in I$. $\left(h_{\alpha}\right)_{\alpha \in I}$ will be called a proper family. If $\left(h_{\alpha}\right)$ is a proper family with the additional properties

(a) $0<h_{\alpha}<$ order of $\bar{x}_{\alpha}$

(b) $x_{\alpha+1}=x_{\alpha}^{-1}$ implies $h_{\alpha+1}=0$ or $h_{\alpha}=0$,

then $\left(h_{\alpha}\right)$ is called a basic family. The set of all proper families is ordered lexicographically, that is, $\left(k_{\alpha}\right)<\left(h_{\alpha}\right)$ if and only if there is an index $\sigma \in I$ such that $k_{\tau}=h_{\tau}$ for all $\tau<\sigma$ and $k_{\sigma}<h_{\sigma}$.

Corresponding to each proper family $\left(h_{\alpha}\right)$ is a proper product:

$$
P\left(h_{\alpha}\right)=\prod_{\alpha}\left(x_{\alpha}-1\right)^{h_{\alpha}}
$$

where the factors occur in increasing order from left to right. If $\left(h_{\alpha}\right)$ is a basic family $P\left(h_{\alpha}\right)$ will be called a basic product. Note that if $h_{\alpha}=0$ for all $\alpha$, $P\left(h_{\alpha}\right)=1$. Using the expression (1) for an element $g \in G$ and the identity

$$
X Y=1+(X-1)+(Y-1)+(X-1)(Y-1)
$$

it can readily be seen that

$$
g=1+e(1)\left(x_{\alpha(1)}-1\right)+\cdots+e(k)\left(x_{\alpha(k)}-1\right)+\cdots,
$$

a linear combination of basic products. Hence, the basic products span $\Gamma$.

Proposition A. The basic products are a Z-basis for $\Gamma$.

Proof. In view of the above remark, it is sufficient to show that the basic products are linearly independent. Suppose $\sum a\left(h_{\alpha}\right) P\left(h_{\alpha}\right), a\left(h_{\alpha}\right) \in Z$, is a linear combination of basic products with at least one $a\left(h_{\alpha}\right) \neq 0$. Among the families $\left(h_{\alpha}\right)$ for which $a\left(h_{\alpha}\right) \neq 0$, there is a maximal one, say $\left(k_{\alpha}\right)$. If we multiply out the products $P\left(h_{\alpha}\right)$ and collect terms, we obtain a linear combination of group elements, each expressed in its unique form (1). But then the element $\prod_{\alpha} x_{\alpha}^{k_{\alpha}}$, the factors occurring in increasing order of $\alpha$ from left to right, occurs with coefficient $a\left(k_{\alpha}\right) \neq 0$. But the elements of $G$ are linearly independent over $Z$. Hence, the given linear combination of basic products is not zero. 
Although an element $\gamma \in \Gamma$ has a unique presentation as a linear combination of basic products, it may, in general, have more than one presentation as a linear combination of proper products. Any such presentation of $\gamma$ will be called a proper presentation. To each proper presentation $\sum a\left(h_{\alpha}\right) P\left(h_{\alpha}\right)$ is assigned an apparent weight $W_{a}\left(\sum a\left(h_{\alpha}\right) P\left(h_{\alpha}\right)\right)$. For a proper product, set

$$
W_{a}\left(P\left(h_{\alpha}\right)\right)=\sum w\left(x_{\alpha}\right) h_{\alpha} ;
$$

for a linear combination of proper products

$$
\begin{aligned}
W_{a}\left(\sum a\left(h_{\alpha}\right) P\left(h_{\alpha}\right)\right) & =\operatorname{Min}\left\{W_{a}\left(P\left(h_{\alpha}\right)\right): a\left(h_{\alpha}\right) \neq 0\right\}, \\
W_{a}(0) & =\infty .
\end{aligned}
$$

The absolute weight $W(\gamma)$ of an element $\gamma$ is defined to be the maximum of the apparent weights of all proper presentations of $\boldsymbol{\gamma}$.

We now define

$$
\Lambda_{i}=\{\gamma \in \Gamma: W(\gamma) \geqq i\}
$$

Proposition B. $\Lambda_{i}$ is a $Z$-module.

Proof. $0 \in \Lambda_{i}$. If $a \neq 0$ is an integer, then $W(a \gamma)=W(\gamma) \geqq i$. If $\gamma$ and $\delta \in \Lambda_{i}$ then each has a proper presentation of apparent weight $\geqq i$; hence, $\gamma+\delta$ has a proper presentation of apparent weight $\geqq i$.

Proposition C. If $\left(g_{1}-1\right)\left(g_{2}-1\right) \cdots\left(g_{k}-1\right)$ is such that $\sum w\left(g_{j}\right) \geqq i$, then this product belongs to $\Lambda_{i}$.

Proof. Since $G_{n+1}=1$, the proposition is true if each $g_{j} \in G_{n+1}$. Assume it is also true if each $g_{j} \in G_{m+1}$. It is sufficient to prove it is true when each $g_{j} \in G_{m}$.

Let

$$
\left(g_{1}-1\right)\left(g_{2}-1\right) \cdots\left(g_{k}-1\right)
$$

be a product with $w\left(g_{j}\right) \geqq m$ for all $j$ and $\sum w\left(g_{j}\right) \geqq i$. Replace each factor $\left(g_{j}-1\right)$ by its presentation as a linear combination of basic products as in equation (2). Since $w\left(g_{j}\right) \leqq w\left(x_{\alpha}\right)$ for each $x_{\alpha}$ in its unique factorization (1), the given product (A) can be written as a sum of products of factors $\left(x_{\alpha}-1\right)$ where $w\left(x_{\alpha}\right) \geqq m$ and, in any product $\sum w\left(x_{\alpha}\right) \geqq i$. Thus, the problem is reduced to consideration of products of the form

$$
Q_{1}\left(x_{\alpha(1)}-1\right) Q_{2}\left(x_{\alpha(2)}-1\right) Q_{3} \cdots Q_{r}\left(x_{\alpha(r)}-1\right) Q_{r+1},
$$

where $Q_{j}$ is a product of factors $z-1$ with $w(z) \geqq m+1$ and where $w\left(x_{\alpha(j)}\right)=m$.

If $r=0$, then (B) belongs to $\Lambda_{i}$ by the induction hypothesis. Assume the proposition is true for all $s<r$. Next, we show that we may write (B) in the form

$$
\left(x_{\alpha(1)}-1\right)\left(x_{\alpha(2)}-1\right) \cdots\left(x_{\alpha(r)}-1\right) Q+\text { elements of } \Lambda_{i}
$$


where $Q$ is a product of factors $z-1$ with $w(z) \geqq m+1$ and where the sum of the weights of the $x_{\alpha(j)}$ and $z$ is $\geqq i$. To do this we apply the identity

$$
\begin{aligned}
(z-1)\left(x_{\alpha}-1\right)= & \left(x_{\alpha}-1\right)\left(x_{\alpha}^{-1} z x_{\alpha}-1\right) \\
& +\left(x_{\alpha}^{-1} z x_{\alpha} z^{-1}-1\right)(z-1)+\left(x_{\alpha}^{-1} z x_{\alpha} z^{-1}-1\right)
\end{aligned}
$$

a sufficient number of times to (B). Since $z \in G_{m+1}, x_{\alpha}^{-1} z x_{\alpha} \in G_{m+1}$ and $x_{\alpha}^{-1} z x_{\alpha} z^{-1}$ $\in G_{2 m+1}$. Therefore, $w\left(x_{\alpha}^{-1} z x_{\alpha}\right) \geqq m+1$ and $w\left(x_{\alpha}^{-1} z x_{\alpha} z^{-1}\right) \geqq 2 m+1$ and so the terms arising from the second and third terms on the right of (I) when (I) is substituted in (B) belong to $\Lambda_{i}$ by the second induction hypothesis. Thus, we need only consider products of the form

$$
\left(x_{\alpha(1)}-1\right)\left(x_{\alpha(2)}-1\right) \cdots\left(x_{\alpha(r)}-1\right) Q,
$$

where $w\left(x_{\alpha(j)}\right)=m$ and $Q$ is a product of factors $z-1$ with $w(z) \geqq m+1$ and where the sum of the weights of the $x_{\alpha(j)}$ and $z$ is $\geqq i$.

Next, we rewrite (C) as a sum of terms of the form

$$
\left(x_{\beta(1)}-1\right)\left(x_{\beta(2)}-1\right) \cdots\left(x_{\beta(r)}-1\right) Q
$$

plus terms of $\Lambda_{i}$, where the factors $x_{\beta(j)}-1$ occur in order of increasing $\beta(j)$ from left to right and conditions as above are satisfied. To accomplish this, apply the identity

$$
\begin{aligned}
(x-1)(y-1)= & (y-1)(x-1) \\
& +(y-1)(x-1)((x, y)-1) \\
& +(x-1)((x, y)-1) \\
& +((x, y)-1)
\end{aligned}
$$

to (C) a sufficient number of times. By the second induction hypothesis, noting that the sum of the weights does not decrease, we observe that the terms arising from the third, fourth and fifth terms on the right of (II) when (II) is substituted in (C) belong to $\Lambda_{i}$. The term arising from the second term of (II) when it is substituted in (C) may be rewritten, as in (C), so that the factor $((x, y)-1)$ lies to the right of all the factors $x_{\alpha(j)}-1$.

Hence, starting with a product (A) which it was desired to show was in $\Lambda_{i}$, we have proved that it is sufficient to consider products of the form

$$
\left(x_{\alpha(1)}-1\right)\left(x_{\alpha(2)}-1\right) \cdots\left(x_{\alpha(r)}-1\right) Q
$$

where each $w\left(x_{\alpha(j)}\right)=m$, the factors $x_{\alpha(j)}-1$ occur in order of increasing $\alpha(j)$, $Q$ is a product of factors $z-1$ with $w(z) \geqq m+1$ and the sum of the weights of the $x_{\alpha(j)}$ and the $z$ is $\geqq i$. We now apply the same sequence of processes to the factors $z-1$ of $Q$ for which $w(z)=m+1$, noting that factors $u-1$ of weight less than $m+2$ are never introduced. We then do the same thing to the factors of weight $m+2$, etc. Since $G_{n+1}=1$, this process terminates in a finite number of steps. The result of the whole procedure, then, is a linear combina- 
tion of proper products all of weight $\geqq i$. Hence, the product (A) is in $\Lambda_{i}$.

Proposition D. $\Lambda_{i} \cdot \Lambda_{j} \subseteq \Lambda_{i+j}$ and, therefore, each $\Lambda_{i}$ is an ideal.

Proof. If $P\left(h_{\alpha}\right)$ and $P\left(k_{\alpha}\right)$ have weights $i$ and $j$, respectively, then $P\left(h_{\alpha}\right) P\left(k_{\alpha}\right)$ has, by Proposition $\mathrm{C}$, weight $\geqq i+j$ and so belongs to $\Lambda_{i+j}$. Since $\Lambda_{0}=\Gamma$, this shows, in particular, that $\Lambda_{i}$ is an ideal

Proposition E. $\Lambda_{i} \supseteq \Delta^{i}$.

Proof. $\Delta^{i}$ is generated by all the products $\left(g_{1}-1\right) \cdots\left(g_{i}-1\right)$. By Proposition $C$, each such product belongs to $\Lambda_{i}$.

Proposition F. $g \in G_{i}$ if and only if $g-1 \in \Lambda_{i}$.

Proof. Let $g \in G_{i}$. Then, by Proposition C, $g-1 \in \Lambda_{i}$.

Conversely, suppose $g-1 \in \Lambda_{i}$. Consider the representation (1) of $g$. To show that $g \in G_{i}$ it is sufficient to show that $x_{\alpha(1)} \in G_{i}$. If it can be shown that every representation of $g-1$ as a linear combination of proper products must include either a nonzero term $a\left(x_{\alpha(1)}-1\right)$ or a nonzero term $a\left(x_{\alpha(1)}^{-1}-1\right)$, $a \in Z$, then $W\left(x_{\alpha(1)}-1\right) \geqq i$ and so $w\left(x_{\alpha(1)}\right) \geqq i$ or $x_{\alpha(1)} \in G_{i}$.

Suppose a given linear combination of proper products is not a linear combination of basic products. Then it must include a term of the form

$$
c Q_{1}\left(x_{\beta}-1\right)\left(x_{\beta}^{-1}-1\right) Q_{2}
$$

or a term of the form

$$
c Q_{1}\left(x_{\beta}-1\right)^{m} Q_{2}
$$

where $m=$ order of $\bar{x}_{\beta}$ and $Q_{2}$ contains no factor $x_{\beta}-1$.

The identity $\left(x_{\beta}-1\right)\left(x_{\beta}^{-1}-1\right)=-\left(x_{\beta}-1\right)-\left(x_{\beta}^{-1}-1\right)$ reduces (1) to

$$
-c Q_{1}\left(x_{\beta}-1\right) Q_{2}-c Q_{1}\left(x_{\beta}^{-1}-1\right) Q_{2} \text {. }
$$

Call the passage from (1) to $\left(1^{\prime}\right)$ operation $I$.

The identity

$$
\begin{aligned}
\left(x_{\beta}-1\right)^{m} & =\left(\begin{array}{c}
m \\
x_{\beta}
\end{array}-1\right)-\sum_{j=1}^{m-1}\left(\begin{array}{c}
m \\
j
\end{array}\right)\left(x_{\beta}-1\right)^{j} \\
& =\left(x_{\beta}^{m}-1\right)-m\left(x_{\beta}-1\right)-\sum_{j=2}^{m-1}\left(\begin{array}{c}
m \\
j
\end{array}\right)\left(x_{\beta}-1\right)^{j}
\end{aligned}
$$

reduces (2) to

$$
-c m Q_{1}\left(x_{\beta}-1\right) Q_{2}-\sum_{j=2}^{m-1} c\left(\begin{array}{c}
m \\
j
\end{array}\right) Q_{1}\left(x_{\beta}-1\right) Q_{2}+c Q_{1}\left(x_{\beta}^{m}-1\right) Q_{2} .
$$

All but the last term are proper products. The last term must be straightened as in the proof of Proposition C. Since $m=\operatorname{order} \bar{x}_{\beta}, w\left(x_{\beta}^{m}\right)>w\left(x_{\beta}\right)$. Since the 
straightening process never introduces terms of lower weight, $c Q_{1}\left(x_{\beta}^{m}-1\right) Q_{2}$ is zero (if $x_{\beta}^{m}=1$ ) or each term of its straightened form contains a factor $x_{\gamma}-1$ with $w\left(x_{\gamma}\right)>w\left(x_{B}\right)$. Thus (2) is replaced by

$$
-c m Q_{1}\left(x_{\beta}-1\right) Q_{2}-\sum_{j=2}^{m-1} c\left(\begin{array}{c}
m \\
j
\end{array}\right) Q_{1}\left(x_{\beta}-1\right)^{j} Q_{2}+R
$$

where $R$ is zero or is a sum of proper products each containing a factor $x_{\gamma}-1, w\left(x_{\gamma}\right)>w\left(x_{\beta}\right)$. Call the passage from (2) to $\left(2^{\prime}\right)$ operation II.

It is clear that any linear combination of proper products can be reduced to a basic linear combination by a finite number of applications of operations I and II.

LEMmA. Let a linear combination of proper products include the terms $a_{1}\left(x_{\beta}-1\right)$ and $a_{2}\left(x_{\beta}^{-1}-1\right)$ where $m$ divides $a_{1}-a_{2}, m=$ order $\bar{x}_{\beta}$. Apply operation I or operation II to any term of the linear combination. Let $b_{1}$ and $b_{2}$ be the new coefficients of $x_{\beta}-1$ and $x_{\beta}^{-1}-1$, respectively, in the resulting linear combination. Then $m$ also divides $b_{1}-b_{2}$.

Proof. (I) The only new terms are

$$
-c Q_{1}\left(x_{\gamma}-1\right) Q_{2} \text { and }-c Q_{1}\left(x_{\gamma}^{-1}-1\right) Q_{2} \text {. }
$$

The coefficients of $x_{\beta}-1$ and $x_{\beta}^{-1}-1$ will be changed if and only if $Q_{1}=Q_{2}=1$ and $x_{\gamma}=x_{\beta}$. In this case, we have $b_{1}=a_{1}-c, b_{2}=a_{2}-c$. Hence,

$$
b_{1}-b_{2}=\left(a_{1}-c\right)-\left(a_{2}-c\right)=a_{1}-a_{2}
$$

and, so, is divisible by $m$.

(II) The only new terms are

$$
-m c Q_{1}\left(x_{\gamma}-1\right) Q_{2}-\sum_{j=2}^{m-1} c\left(\begin{array}{c}
m \\
j
\end{array}\right) Q_{1}\left(x_{\gamma}-1\right)^{i} Q_{2}+R .
$$

The coefficients of $x_{\beta}-1$ and $x_{\beta}^{-1}-1$ will be changed if and only if $Q_{1}=Q_{2}=1$ and $x_{\gamma}=x_{\beta}$ or $x_{\gamma}=x_{\beta}^{-1}$. Hence, we will have either

$$
b_{1}=a_{1}-m c, \quad b_{1}=a_{1},
$$

or

$$
b_{2}=a_{2}, \quad b_{2}=a_{2}-m c .
$$

Therefore, $b_{1}-b_{2}=a_{1}-a_{2} \pm m c$ is divisible by $m$.

Now, let us suppose that there is a proper presentation of $g-1$ which does not include a nonzero multiple of $x_{\alpha(1)}-1$ or a nonzero multiple of $x_{\alpha(1)}^{-1}-1$; in terms of the lemma, $a_{1}=a_{2}=0$. Then, if $m=$ order of $\bar{x}_{\alpha(1)}, m$ divides $a_{1}-a_{2}$. By applying operations I and II to bring this representation of $g-1$ to the basic form (2), we find that we must have $m$ dividing $e(1)$. But $0<e(1)$ 
$<m$. Hence, no such proper presentation exists and so, by the above remarks, Proposition $F$ is proved.

The proof of Theorem 5.1 is now complete for the case $G_{n+1}=1$. Let us now drop this assumption.

Consider the natural homomorphism

$$
\phi_{n}: G \rightarrow G^{(n)}=G / G_{n+1}
$$

and its extension to $\Gamma$,

$$
\Phi_{n}: \Gamma \rightarrow \Gamma_{n}=\Gamma / I\left(G_{n+1}\right) \text {. }
$$

The images of the $G_{i}$ under $\phi_{n}$ are just the commutator subgroups $G_{i}^{(n)}$ of $G^{(n)}$ and $G_{n+1}^{(n)}=1$. For each $n$ construct the sequence of ideals $\Lambda_{n, i}$ having the properties

(a) $g \in G_{i}^{(n)}$ if and only if $g-1 \in \Lambda_{n, i}$,

(b) $\Lambda_{n, 2} \supseteq \Delta_{n}^{i}$, where $\Delta_{n}$ is the fundamental ideal of $\Gamma_{n}$.

We now set

$$
\Lambda_{i}=\bigcap_{n} \Phi_{n}^{-1}\left(\Lambda_{n, i}\right)
$$

$\Lambda_{i}$ is the intersection of ideals and, thus, itself an ideal. If $g \in G_{i}$ then $\phi_{n}(g)$ $\in G_{i}^{(n)}$ and so $\phi_{n}(g-1)=\phi_{n}(g)-1 \in \Lambda_{n, i}$ for every $n$. Therefore $g-1 \in \Lambda_{i}$. Conversely, if $g-1 \in \Lambda_{i}$ then $\bar{\phi}_{n}(g-1)=\phi_{n}(g)-1 \in \Lambda_{n, i}$ and, hence, $\phi_{n}(g) \in G_{i}^{(n)}$ for every $n$. Consequently, $g \in G_{i}$. Furthermore, it is clear that $\phi_{n}\left(\Delta^{i}\right) \subseteq \Lambda_{n, i}$ for every $n$ and, thus, that $\Delta^{i} \subseteq \Lambda_{i}$. This completes the proof of Theorem 5.1.

THEOREM 5.2. If $G$ is any group and $\left\{D_{i}\right\}$ the dimension series $\bmod Z$ of $G$, then $D_{n}=G_{n}$ for every n.(2)

Proof. It has already been shown that $G_{n} \subseteq D_{n}$. Now let $g \in D_{n}$. Then $g-1 \in \Delta^{n}$ and so

$$
g-1=\sum \alpha_{i}\left(g_{i 1}-1\right) \cdots\left(g_{\text {in }}-1\right) .
$$

Let $H$ be the subgroup of $G$ generated by all the $g_{i j}$ for which $\alpha_{i} \neq 0$. $H$ is finitely generated. Therefore

$$
g \in D_{n}(H ; Z)=H_{n} \subseteq G_{n} .
$$

Consequently, $D_{n} \subseteq G_{n}$ and, thus, $D_{n}=G_{n}$.

THEOREM 5.3. If $\Gamma$ is the group ring of $G$ over the ring of integers, then

$$
G_{n} / G_{n+1}=\left(\Delta^{(n)}+\Delta^{n+1}\right) / \Delta^{n+1} \text {. }
$$

(2) The author wishes to thank the referee for pointing out that the general result follows from the result for finitely generated groups. 
Corollary 5.4. Let $G$ and $H$ be groups. Then $G$ and $H$ have isomorphic group rings over the integers only if the factors of their lower central series are isomorphic.

As our final result, we indicate the proof of a theorem generalizing a result of Jennings [4]. Note that the ideals $\Lambda_{i}$ constructed in the proof of Theorem 5.1 must, in fact, be precisely the $\Delta^{i}$. If $G$ is a finitely generated nilpotent group then each element of $\Gamma$ must have a definite finite weight $i$ and, hence, will not belong to $\Delta^{i+1}$. Thus,

THEOREM 5.5. If $G$ is a finitely generated nilpotent group and $\Gamma$ the group ring of $G$ over $Z$, then

$$
\bigcap_{i} \Delta^{i}=(0)
$$

\section{REFERENCES}

1. P. M. Cohn, Generalization of a theorem of Magnus, Proc. London Math. Soc. vol. 57 (1952) pp. 297-310. See correction in the same volume.

2. R. H. Fox, Free differential calculus. I, Ann. of Math. vol. 57 (1953) pp. 547-560.

3. M. Hall, Theory of groups, New York, Macmillan, 1959.

4. S. A. Jennings, The group ring of a class of infinite nilpotent groups, Canad. J. Math. vol. 7 (1955) pp. 169-187.

5. - The structure of the group ring of a p-group over a modular field, Trans. Amer. Math. Soc. vol. 50 (1941) pp. 175-185.

6. M. Lazard, Sur les groupes nilpotents et les anneaux de Lie, Thesis, Paris, 1954.

7. W. Magnus, Beziehungen zwischen Gruppen und Idealen in einem speziellen Ring, Math. Ann. vol. 111 (1935) pp. 259-280.

8. H. Zassenhaus, Ein Verfahren, jeder endlichen p-Gruppe einen Lie-Ring mit der Charackteristik p zuzuordnen, Abh. Math. Sem. Hamburg Univ. vol. 13 (1939) pp. 200-207.

UNIVERSITY OF WISCONSIN, Madison, Wisconsin 